\title{
Correction to: Self-Directed Gambling Changes: Trajectory of Problem Gambling Severity in Absence of Treatment
}

\author{
Vladyslav Kushnir ${ }^{1,2}$ - Alexandra Godinho ${ }^{1,3}$ • David C. Hodgins ${ }^{4}$. \\ Christian S. Hendershot ${ }^{1,5,6} \cdot$ John A. Cunningham ${ }^{1,6,7}$
}

Published online: 19 May 2018

(C) Springer Science+Business Media, LLC, part of Springer Nature 2018

\section{Correction to: J Gambl Stud \\ https://doi.org/10.1007/s10899-018-9769-8}

The original version of this article unfortunately contained errors in the Methods section. The citations blinded during the review process were inadvertently omitted during the production process and subsequent stages.

The incorrect reference citations in Participants section and Study Design and Procedures section have been corrected and the below Reference is added.

Kushnir, V., Cunningham, J. A., \& Hodgins, D. C. (2013). A prospective natural history study of quitting or reducing gambling with or without treatment: Protocol. JMIR Research Protocols, 2(2), e51. https://doi.org/10.2196/resprot.2727.

The original article has been corrected.

The original article can be found online at https://doi.org/10.1007/s10899-018-9769-8.

Vladyslav Kushnir

vlad.kushnir@mail.utoronto.ca

1 Institute for Mental Health Policy Research, Centre for Addiction and Mental Health, 33 Russell

St, Toronto, ON M5S 2S1, Canada

2 Leslie Dan Faculty of Pharmacy, University of Toronto, Toronto, Canada

3 Dalla Lana School of Public Health, University of Toronto, Toronto, Canada

4 Department of Psychology, University of Calgary, Calgary, Canada

5 Campbell Family Mental Health Research Institute, Centre for Addiction and Mental Health, Toronto, Canada

6 Department of Psychiatry, University of Toronto, Toronto, Canada

7 Research School of Population Health, Australian National University, Canberra, Australia 